\title{
FATIGUE CRACK INITIATION IN A CARBON BLACK-FILLED NATURAL RUBBER
}

\author{
Bertrand Huneau, ${ }^{1, *}$ ISAure Masquelier, ${ }^{2}$ YAnn Marco, ${ }^{2}$ Vincent Le SAuX, ${ }^{2}$ Simon Noizet,${ }^{3}$ \\ Clémentine SCHIEl, ${ }^{3}$ PIERRE CHARRIER ${ }^{3}$

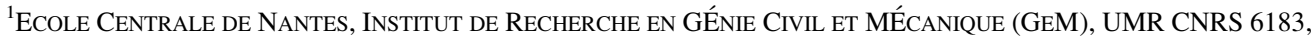 \\ NANTES, FRANCE \\ ${ }^{2}$ ENSTA Bretagne, Laboratoire Brestois de MÉcAnique des Structures (LBMS), EA 4325, Brest, FranCE \\ ${ }^{3}$ TrelleborgVibracoustic Group, CAE Durability PREDICTION DEPARTMENT, CARQuEFOU, France
}

\begin{abstract}
A detailed study of the initiation of fatigue cracks in carbon black-filled natural rubber is conducted. Interrupted fatigue tests are performed and fatigued samples are observed with a scanning electron microscope. This procedure first enables the quantification of the morphology, spatial distribution, and evolution of crack initiation sites for different strain levels, which gives some statistical data for each strain level. It also permits analysis of the chemical nature of inclusions inducing crack initiation thanks to energy dispersive spectrometry of X-rays. It is shown that fatigue damage initially occurs generally on carbon black agglomerates or oxides such as $\mathrm{ZnO}$. However, those two types of inclusions correspond to different crack initiation mechanisms, and most of the time, only the initiations on carbon black agglomerates are followed by crack propagation that leads to failure. This difference is probably because carbon black agglomerates have a stronger cohesion than $\mathrm{ZnO}$ inclusions and a stronger adhesion to the matrix.
\end{abstract}

\section{INTRODUCTION}

The total life of industrial rubber parts subjected to fatigue can be decomposed in two phases: crack initiation and crack propagation. Consequently, two approaches are generally considered to analyze and to predict the fatigue life of rubber: the crack initiation (nucleation) approach and the crack propagation approach, as proposed by Mars and Fatemi. ${ }^{1}$

The knowledge of the size of initial crack/flaws (generally denoted $\mathrm{a}_{0}$ or $\mathrm{c}_{0}$ ) is particularly important for the crack propagation approach because it explicitly considers preexisting cracks or flaws. ${ }^{1}$ Moreover, the integration of the propagation law requires this value and allows for the prediction of fatigue life, as originally shown by Gent et al. ${ }^{2}$ But what really represents this initial flaw size at a microstructural scale? From the early studies on rubber fatigue, the initial flaws responsible for initiation of fatigue cracks are generally considered inhomogeneities, as called by Beatty ${ }^{3}$ reminiscent of the seminal concept of "flaws" proposed by Griffith to explain the mechanical weakness of structural materials. ${ }^{4}$ For filled natural rubber (NR), which exhibits excellent fatigue properties, ${ }^{3,5-7}$ these inhomogeneities can be filler agglomerates (silica or carbon black $[\mathrm{CB}]$ ) or oxides, such as zinc oxide..$^{8-11}$ The size of these inclusions ranges from a few microns to a few hundreds of microns. This was confirmed in a previous study in which the damage of a sample was followed throughout its fatigue life. ${ }^{12}$ However, the precise damage mechanisms leading to the initiation have not been well understood. A recent study has allowed us to clarify this point. $^{13}$

The damage induced by fatigue loading in rubber can be investigated by different experimental techniques. One can cite relatively recent techniques in the field of materials characterization such as X-ray computed microtomography ${ }^{12,14,15}$ but also a more classical observation technique: scanning electron microscope (SEM). SEM remains indeed a powerful tool to observe either fatigue fracture surfaces ${ }^{11,16}$ or interrupted fatigued samples to acquire more precise information about the 
TABLE I

CHEMICAL COMPOSITION OF THE NR43 COMPOUND

\begin{tabular}{lc}
\hline Constituent & Amount, phr \\
\hline NR (SMR 5CV 60) & 100 \\
Carbon black (CB) & 43 \\
Zinc oxide & 5 \\
Sulfur & 1.8 \\
CBS & 2.5 \\
Stearic acid & 2 \\
Plasticizer (Nytex 860) & 3 \\
IPPD & 1 \\
TMQ & 1 \\
\hline
\end{tabular}

mechanisms of crack initiation and crack propagation. ${ }^{8,17-20}$ Nevertheless, these studies rarely focus only on crack initiation, probably because this phenomenon is not easily predictable in terms of location (where the crack is initiated) or time (after how many cycles).

The first objective of the present study is to obtain some statistical information about fatigue crack initiation sites: location, distribution, nature, and evolution, by SEM observations of the whole damaged surface of the samples at different moments in the fatigue life. The second objective is to describe the fatigue crack initiation mechanisms for the two main types of inclusions responsible for it.

\section{EXPERIMENTAL PROCEDURE}

\section{MATERIALS AND SAMPLES}

The investigated elastomer is an NR filled with CB and vulcanized with sulfur. The chemical composition is given in Table I. Most of the results presented here are obtained on an NR filled with CB N550 type, but some results presented in the section "Fatigue Crack Initiation Mechanism for CB Agglomerates" are obtained with other CB types: N220, N326, N375, and N772. For the latter type, the $\mathrm{CB}$ amount is raised to $50 \mathrm{phr}$ in order to have a stiffness comparable with one of the other materials.

In the following, the material's designation refers to its $\mathrm{CB}$ content and $\mathrm{CB}$ type. For example, the main studied compound is referred to as NR43-N550.

\section{FATIGUE TESTS}

Fatigue tests are performed at Trelleborg Vibracoustic on a specific device on hourglassshaped specimens (referred to as AE2 in the following). AE2 geometry is shown in Figure 1a; the median zone of those specimens is $10 \mathrm{~mm}$ in diameter. Because of this particular geometry, the strain field is very heterogeneous. The maximum strains are reached at the surface of the sample and are calculated by finite element analysis (FEA). Figure $1 \mathrm{~b}$ is an example of an FEA result showing the maximum principal strain field for a local maximum value of $100 \%$. Fatigue results are plotted with the local maximum strain in the median zone (slice 1, Figure 2). The lower strains corresponding to slices $2 / 2^{\prime}$ and $3 / 3^{\prime}$ will be considered to investigate the influence of strain level on the number of initiation sites (Figure 2).

Fatigue tests are monitored with prescribed displacement and for a minimum displacement equal to $0 \mathrm{~mm}$. No mechanical accommodation is performed on samples before fatigue tests. The 
a

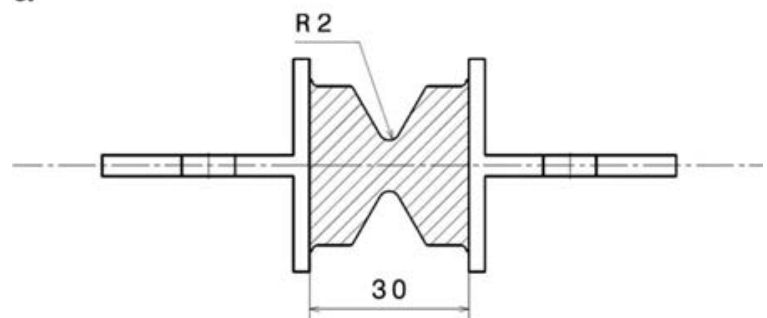

b
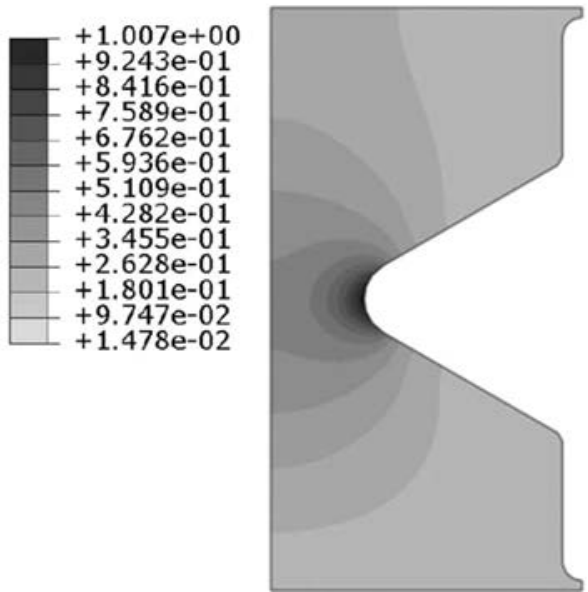

FIG. 1. - (a) Geometry of the AE2 sample. (b) FEA giving the maximum principal strain field for a local maximum strain of $100 \%$.

frequency of the tests varies from $5 \mathrm{~Hz}$ to $1 \mathrm{~Hz}$ in order to limit heat buildup. By using 25 specimens, a Wöhler curve, shown in Figure 3a, is plotted with the maximum strain value (at the surface of slice 1 ) as a function of the number of cycles to the fatigue end of life of the specimens. This number is called $\mathrm{Ni}$ and is detected by a loss of specimen stiffness (see Ostoja-Koczynski et al. ${ }^{21}$ for details). The Wöhler curve is used as a reference to define the strain level and the number of cycles for each sequence of the interrupted fatigue tests. Figure $3 \mathrm{~b}$ shows a typical evolution of the maximum force during the fatigue tests with the number of cycles. $\mathrm{Ni}$, as well as the three percentages of fatigue life $(20 \% \mathrm{Ni}, 40 \% \mathrm{Ni}$, and $60 \% \mathrm{Ni}$ ) considered in the present study, are plotted on this curve.

In a previous study, ${ }^{12}$ we investigated the damage evolution along the fatigue life by following the same sample. Here, the following protocol is chosen for the interrupted fatigue tests: each

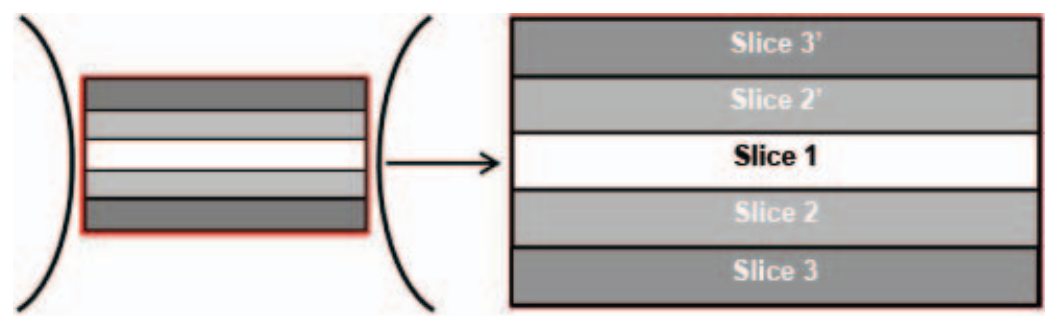

FIG. 2. - Observed zone and definition of the different slices. 


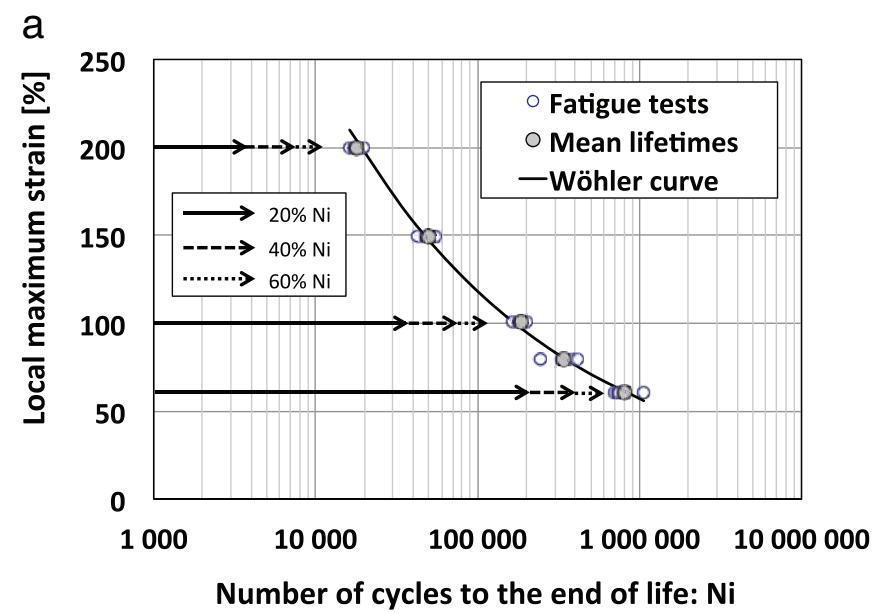

b

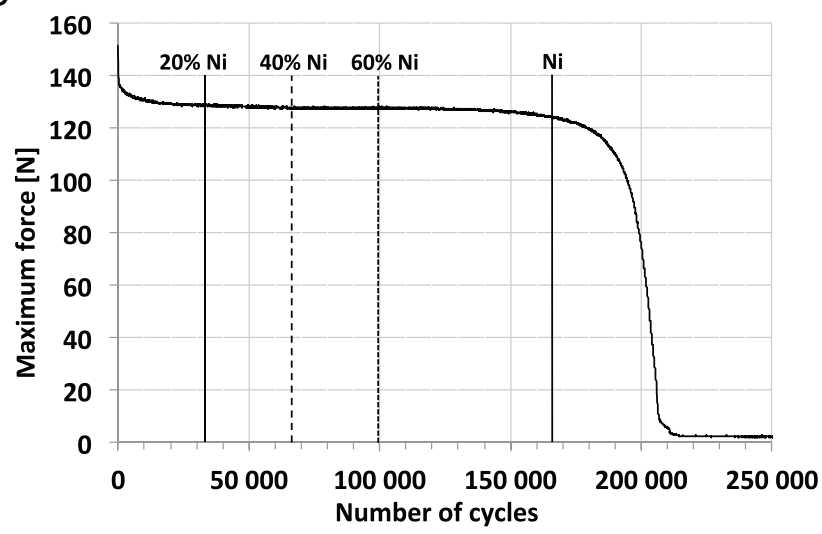

FIG. 3. - (a) Wöhler curve obtained on NR43-N550 for the three local maximum strain levels and the three percentages of fatigue life $(\% \mathrm{Ni})$. (b) Evolution of the maximum force versus the number of cycles with the three percentages of fatigue life.

sample is subjected to one of the three strain levels $(60 \%, 100 \%$, and $200 \%)$ and to one of the three percentages of fatigue life $(20 \% \mathrm{Ni}, 40 \% \mathrm{Ni}$, and $60 \% \mathrm{Ni})$. Consequently, nine samples were observed for NR43-N550 and 12 additional samples for the complementary tests on the influence of CB type. This particular protocol is chosen to have statistical information and to avoid the cumulative damage that can be caused by the electron beam of the SEM, when one follows the same sample. Finally, the very low scattering of fatigue lives for the considered NR (see Figure 3a) justifies this protocol.

\section{SEM OBSERVATIONS AND ENERGY DISPERSIVE SPECTROMETER ANALYSIS}

The SEM investigations are performed with a JEOL 6060-LA. The acceleration voltage is 20 $\mathrm{kV}$, and the images are taken by using the secondary electrons signal. The acceleration voltage was chosen to be relatively high when one considers observation of elastomers. However, thanks to the relatively high $\mathrm{CB}$ content, the material is conductive enough to support his voltage. The previously described procedure consisting in observing each sample only once also justified the use of such a high voltage, which could be involved in fatigue life reduction in the case of multiple observations 


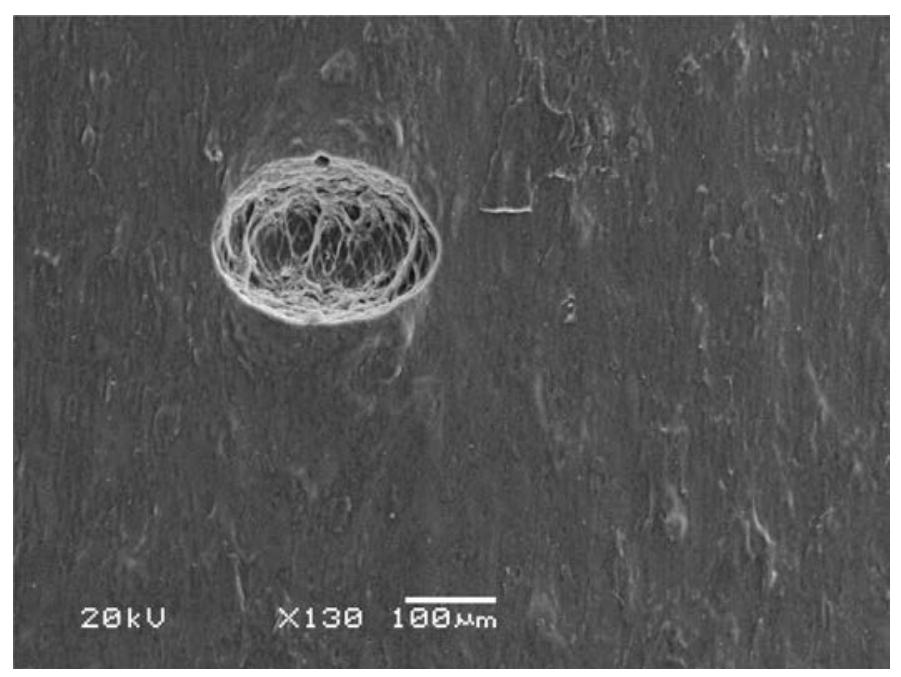

FIG. 4. - Typical SEM image at $\times 130$ magnification with one fatigue crack.

on the same sample. The chemical composition of inclusions is determined thanks to an energy dispersive spectrometer (EDS) of X-rays. Thanks to the use of the high acceleration voltage, the detection of the high atomic number element is facilitated.

Observations are performed on fatigued samples stretched by $3 \mathrm{~mm}$ by using four polymethyl methacrylate beams. The observed zone, which is $3.75 \mathrm{~mm}$ in height and $6.9 \mathrm{~mm}$ in width, is analyzed throughout the five slices defined in Figure 2. To observe the whole circumference of the samples, this operation is repeated four times, after successive rotations of $90^{\circ}$. For one sample, 140 SEM images are taken (seven per slices $\times$ five slices $\times$ four sides), which makes 1260 SEM images for NR43-N550 (140 images $\times$ nine samples). The magnification is $\times 130$ for each image, which enables the detection of inclusions larger than $5 \mu \mathrm{m}$. Figure 4 gives an example of one of these images, which were used for the statistical analysis. Higher magnification SEM images are performed when necessary.

\section{RESULTS AND DISCUSSION}

\section{DAMAGE MECHANISMS IN FATIGUE}

For the considered experimental conditions and elastomers, the fatigue loading always induces a multicracking phenomenon. The numerous cracks observed are initiated either at the parting line or out of the parting line, as illustrated in Figure 5. This was first observed on fracture surfaces and then confirmed by interrupted fatigue tests. These interrupted tests also reveal that initiation of cracks starts very early in fatigue life and is followed by a relatively slow propagation. The multiinitiation and the low fatigue crack growth rates can partly explain the very low scattering obtained for fatigue lives (Figure 3a).

\section{INFLUENCE OF THE NUMBER OF CYCLES AND OF THE MAXIMUM STRAIN}

The density of surface cracks (only cracks longer than $5 \mu \mathrm{m}$ are considered here) is plotted in Figure 6 for the three percentages of the fatigue life $\mathrm{Ni}(20 \%, 40 \%, 60 \%)$ and for the three maximum strain values: $60 \%, 100 \%, 200 \%$. These maximum values are related to the observations made for slice 1 (Figure 2). In addition, the results obtained in slices $2 / 2^{\prime}$ and in slices $3 / 3^{\prime}$ are plotted with 


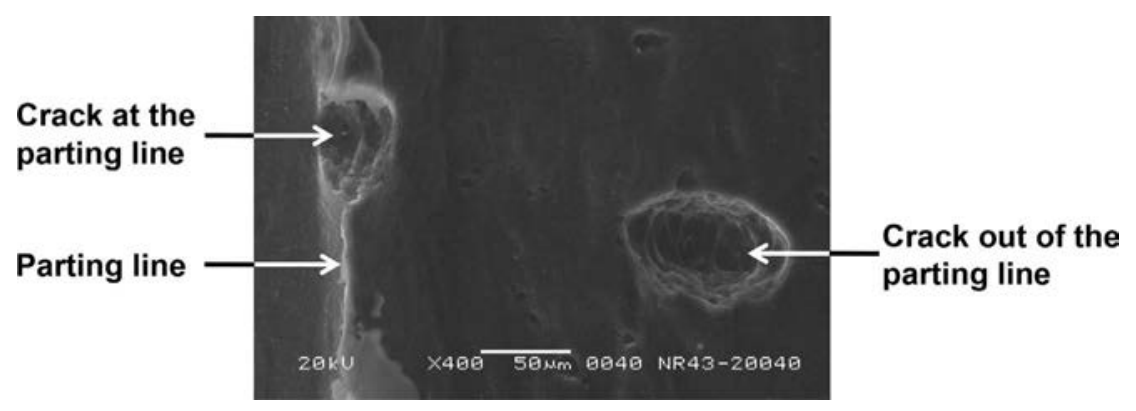

FIG. 5. - SEM image of the typical locations of fatigue crack initiation sites: at the parting line or out of the parting line.

their corresponding strain calculated by FEA. Figure 6 clearly illustrates that the density of cracks increases with the percentage of fatigue life, which means that new crack initiation sites are activated all along the fatigue life. As expected, the density of cracks also increases with the strain level.

\section{NATURE OF THE INCLUSIONS AND CRITICALITY}

As mentioned previously, the cracks are initiated either at the parting line, which can be considered as a geometrical defect acting as a stress concentrator, or around inclusions. In the following, the attention is focused only on cracks initiated on inclusions.

Thanks to EDS, it was possible to analyze the chemical nature of those inclusions, which are sorted here in five categories. The first two categories correspond to CB agglomerates (denoted as "Carbon Black") and to large $\mathrm{ZnO}$ particles (between 2 and $5 \mu \mathrm{m}$ ), denoted as "ZnO." The third category is related to "Talc," and the fourth concerns all the "other oxides," such as calcium/silicon oxides (" $\mathrm{SiO}_{2}$," "CaO") or oxides containing aluminum and silicon (probably kaolinite, referred to as "Al-Si-O" in the following). All those "other oxides" can be introduced into the materials by the NR gum that contains some impurities, the covering powder of the sulfur, and CBS pellets or the process (minor pollution due to a previous mixing/injection, for example). Sometimes, it was not possible to clearly see the inclusions related to the origin of a crack. This could be due to the low magnification used in this study or simply to the absence of inclusions. In the latter case, it should be noted that inclusions could be lost during the sample manipulation. Finally, a fatigue crack initiation

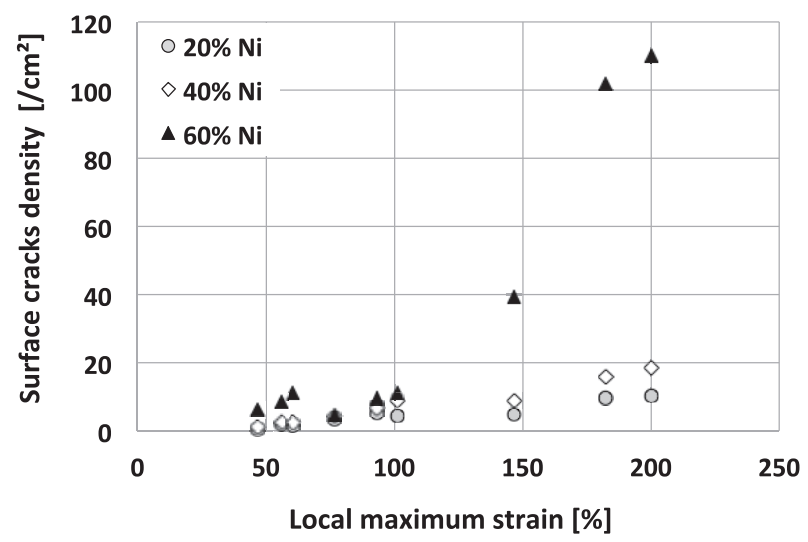

FIG. 6. - Density of surface cracks with respect to the local maximum strain for the three percentages of fatigue life (\% Ni). 


\section{a}
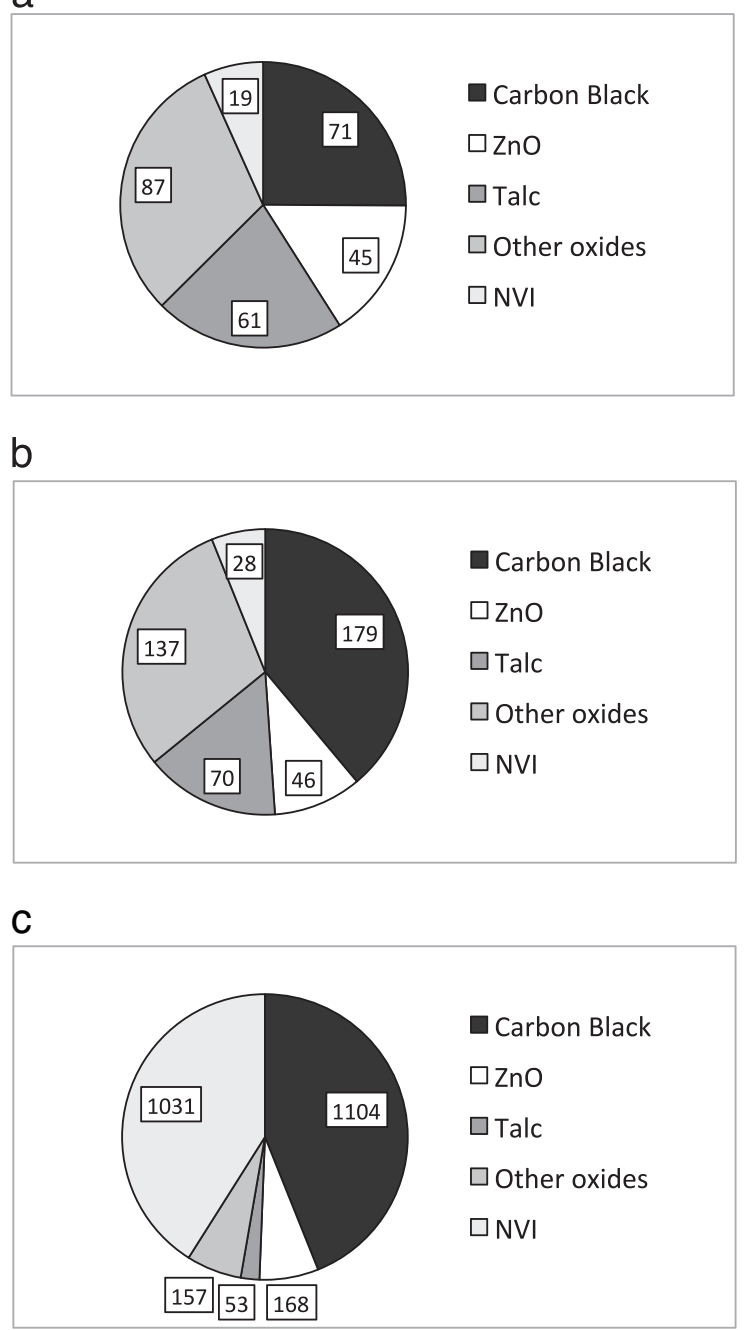

FIG. 7. - Distribution of the different categories of inclusions at the origin of fatigue damage: (a) at $60 \%$ strain, (b) at $100 \%$ strain, (c) $200 \%$ strain.

due to a microgeometrical defect related to the roughness of the mold is also likely. The fifth category is thus called "nonvisible inclusions."

Figure $7 \mathrm{a}-\mathrm{c}$ corresponds to the distribution, in number, of these five categories for the three considered maximum strain levels: $60 \%, 100 \%$, and $200 \%$. For the highest strain level, CB clearly appears to be the most common inclusion, but for the two lowest strain levels, no type of inclusion seems to be majoritarian: the number of inclusions identified as being $\mathrm{CB}, \mathrm{ZnO}$, talc, or other oxides are in the same order of magnitude. Thus, it appears necessary to investigate the relationship between the chemical nature of the inclusions and their criticality in terms of long cracks contributing to the fatigue failure.

To perform such an analysis, the size of the cracks is compared with the size of the inclusions for the four kinds of inclusions listed before ("nonvisible inclusions" are not considered in the following) and for the three strain levels. The results are shown in Figure 8a-c. On these three 
a

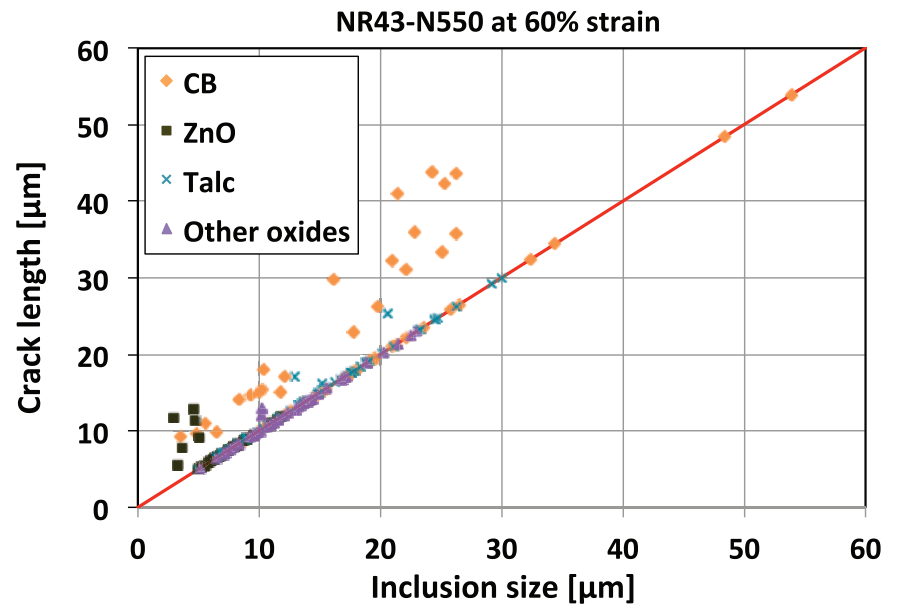

b

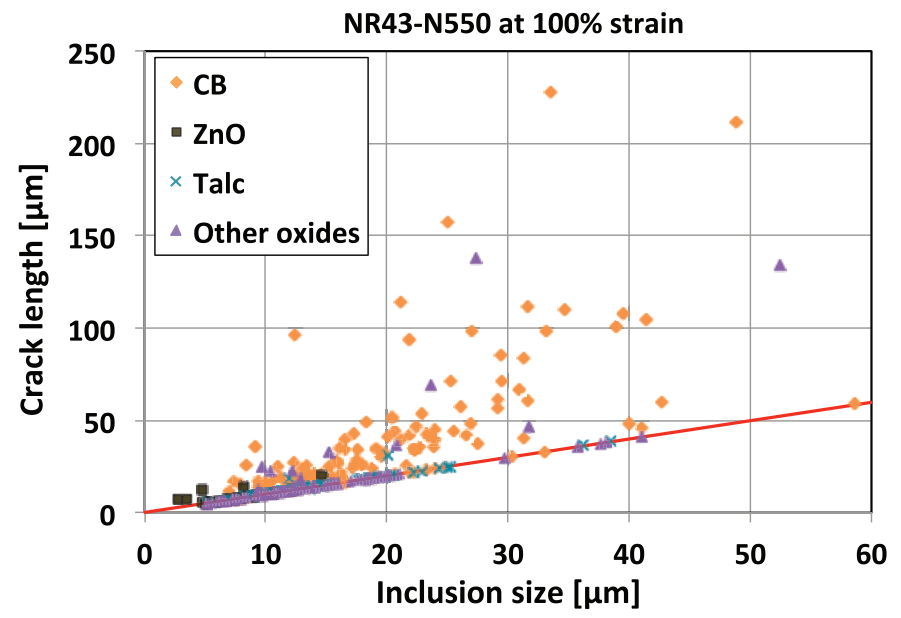

C

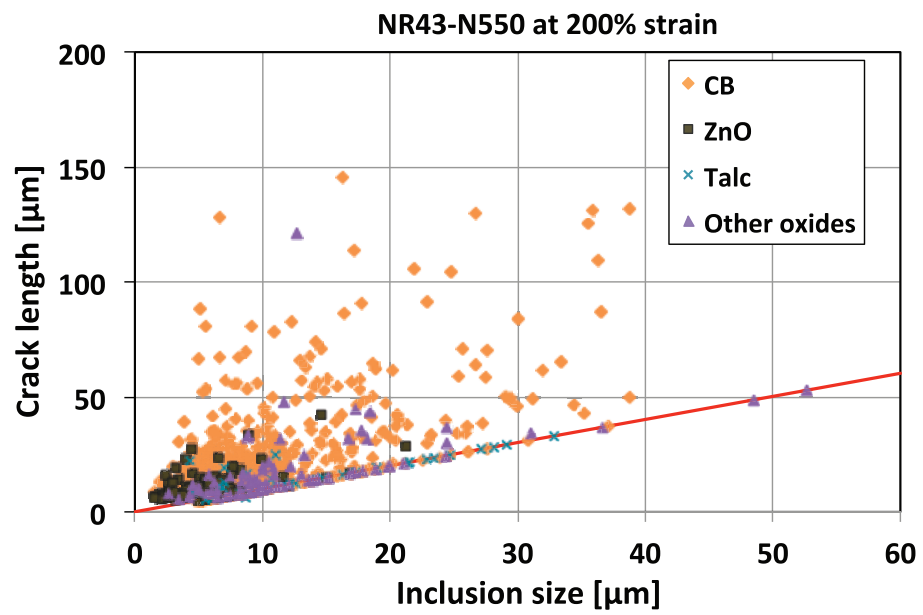

FIG. 8. - Size of the cracks as the function of the size of the inclusions for the three strain levels: (a) $60 \%$, (b) $100 \%$, (c) $200 \%$. 


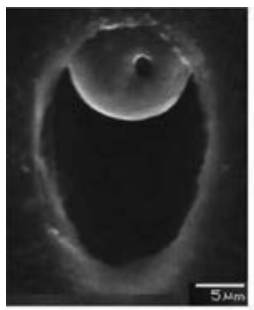

Stage 1

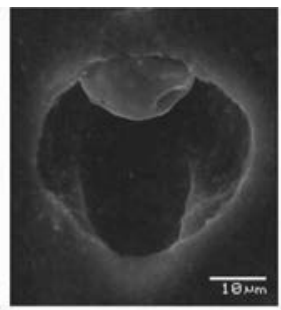

Stage 2

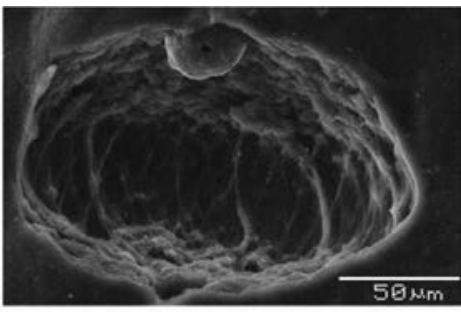

Stage 3

FIG. 9. - The three stages of fatigue crack initiation around a CB agglomerate: debonding at one pole (stage 1), opening on the sides (stage 2), growth at the surface and in the volume (stage 3).

graphs, the line (having a slope of 1) corresponds to sites that do not give rise to the propagation of a crack, as the crack length is almost equal to the size of the inclusion. In that case, the sites are considered "activated": there is some visible damage around the inclusion (cavity), but the size of the cavity is not bigger than the size of the inclusion. Critical cracks are therefore those whose length is much higher than the size of the inclusion responsible for its initiation. From Figure 8, it can be seen that for the three strain levels, CB agglomerates are the inclusions leading to the longer cracks; those that are initiated by the other types of inclusions propagate only a little or not at all, except for Talc and Other oxides, which very rarely lead to the formation of long cracks. Moreover, it can be noted that the relatively high number of activated sites is due to $\mathrm{ZnO}$ inclusions, but they never lead to a crack longer than a few tens of micrometers. This kind of inclusion appears to be the less deleterious, which could be due to its relatively small size, most being smaller than $10 \mu \mathrm{m}$. But this is probably not the only reason, because some CB agglomerates smaller than $20 \mathrm{~mm}$ lead to long cracks, especially at $200 \%$ strain.

The initiation mechanisms of fatigue cracks due to the most harmful inclusions (i.e., $\mathrm{CB}$ ) and to the less harmful ones (i.e., $\mathrm{ZnO}$ ) are more deeply investigated in the next section, in order to better understand the cause of this difference. We will also try to find out what drives the initiation: a critical mechanical quantity and/or energy accumulation?

\section{FATIGUE CRACK INITIATION MECHANISM FOR CB AGGLOMERATES}

From the analysis of the SEM images, the fatigue crack initiation mechanism around CB agglomerates can be divided into three stages represented in Figure 9:

- Stage 1: debonding at the pole

- Stage 2: opening on the sides

- Stage 3: growth at the surface and in the volume, which can be also considered as the early stages of crack propagation

Note that these three SEM pictures are related to three different inclusions.

Stages 1 and 2 are associated with the initiation of the crack and are driven by the inclusion and its interface with the matrix, whereas the matrix controls stage 3 , with no influence of the inclusion. Cracks in stage 3 show some ligaments at the crack tip, which is in agreement with the literature. ${ }^{17,19}$ Figure 10 illustrates schematically the initiation mechanism from the initial situation, which can be called stage 0 , to the propagation (stage 3 ).

CB agglomerates observed in the present study seem to have high internal cohesion (no broken agglomerates were observed) and good interface properties (i.e., a good adhesion to the matrix). The initiation mechanism occurs here by debonding, showing that the internal cohesion of the 


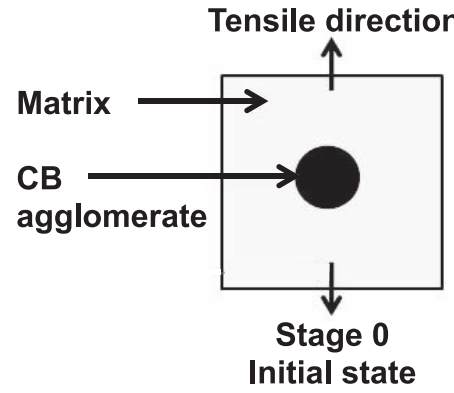

FIG. 10. - Schematic representation of fatigue crack initiation mechanism around a CB agglomerate.

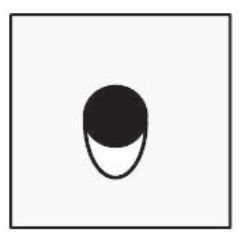

Stage 1

Debonding at

the pole

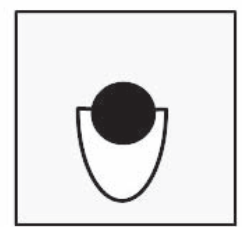

Stage 2

Opening on the

sides

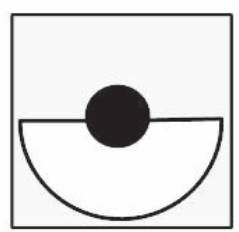

Stage 3

Crack

Propagation

agglomerates is stronger than the filler-matrix interface. We remark also that all CB agglomerates exhibit a spherical shape. It suggests that, during the mixing, the CB pellets could be first broken and then eroded, producing the spherical shape. Those two mechanisms are classically involved in the dispersion of $\mathrm{CB} .^{22}$ To see the internal structure and composition of such agglomerates, one of them has been collected and fractured between two small glass slides used for optical microscopy. Figure 11a shows a CB agglomerate before fracture, and Figure $11 \mathrm{~b}$ shows the same agglomerate after fracture. Besides the fact that this fracture is very brittle (suggesting no or little occluded rubber), it appears to be homogeneous in terms of appearance and carbon content (see the EDS map of carbon in Figure 11c). Consequently, CB agglomerates do not seem to be covered by a layer of rubber. This would confirm a debonding mechanism rather than cavitation in the matrix.

A complementary analysis is then performed to go deeper into the understanding of the proposed mechanism: it consists of classifying the flaws due to fatigue into the three previous stages and for the three percentages of the fatigue life. A flaw can be an activated site or a crack. As shown in Figure 12a, at $100 \%$ strain, the percentage of flaws in stage 1 decreases during the fatigue life, whereas the percentages of flaws in stages 2 and 3 increase. These evolutions, also observed at $200 \%$ strain (see Figure 12b), tend to validate the proposed mechanism, in so far as it is progressive, and it confirms that the CB agglomerates are responsible for the critical cracks (stage 3).

In addition, to determine whether initiation is driven by an accumulation of energy and/or requires a critical mechanical quantity, the minimal size of $\mathrm{CB}$ agglomerates generating initiation is followed for each stage and reported in Table II for the different strain levels and fatigue life percentages. In this table, it can be seen, on one hand, that for stages 2 and 3 and for a given strain level, the minimum size of the flaws generally decreases when the percentage of fatigue life increases. This suggests that the transitions from stage 1 to 2 and from stage 2 to 3 require an accumulation of energy: the inclusions involved in the different damage stages are progressively smaller along the fatigue life. The cracks associated with these inclusions need a given number of cycles, that is, a certain amount of energy (cumulative damage?), to be created. On the other hand, for stages 2 and 3, for the same percentage of fatigue life, the minimum size always decreases when the strain level increases, suggesting that a critical mechanical value has to be reached to create cracks and to start propagating them.

Consequently, it seems that the different stages of the initiation mechanism involving $\mathrm{CB}$ agglomerates require both energy accumulation and a critical mechanical value.

Finally, the question arises regarding the influence of the average size of CB agglomerates (on which cracks are initiated) on the fatigue lifetime. To consider this question, an additional study has been conducted by using NR filled with four other CB types, as mentioned in the section "Materials and Samples." Figure 13 reports the fatigue end of life at $100 \%$ strain with respect to the average 

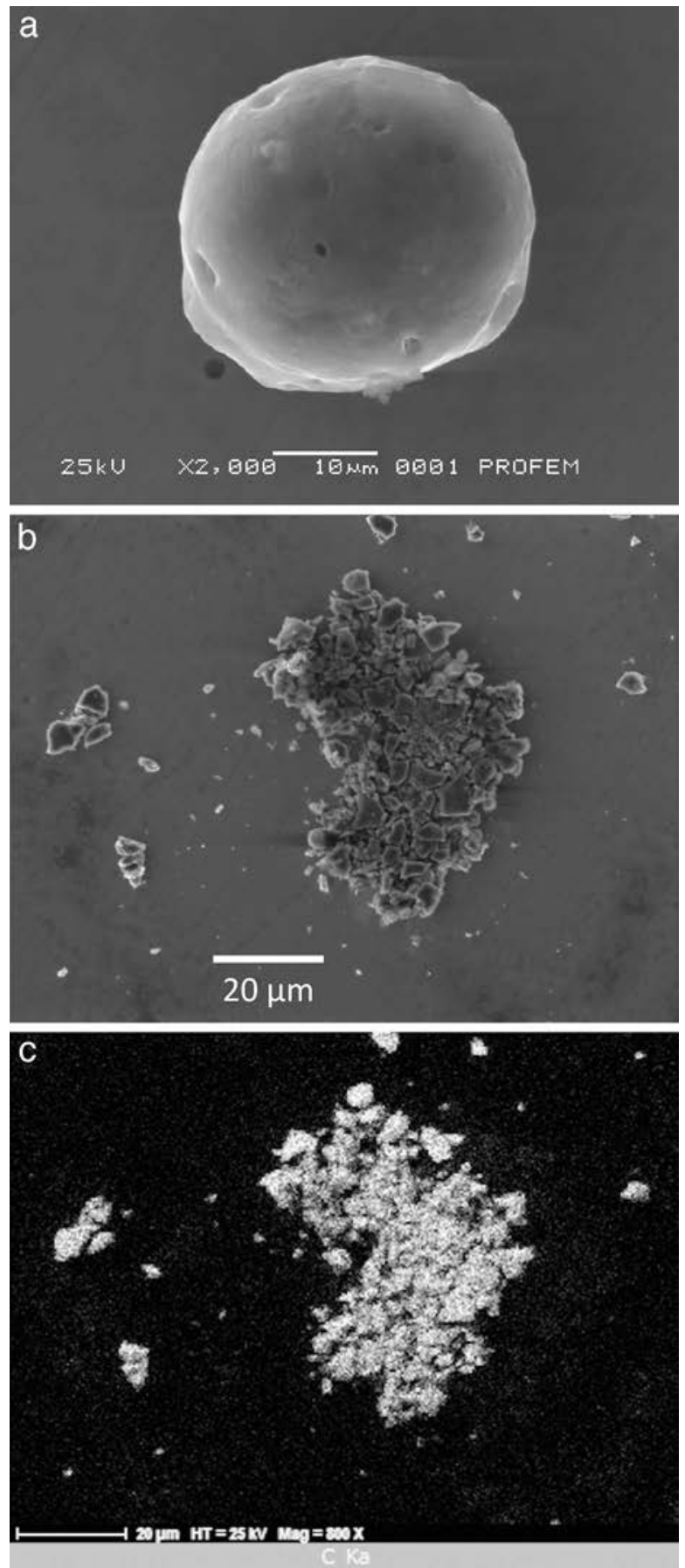

FIG. 11. - A carbon black agglomerate (a) before and (b) after fracture. (c) EDS map of carbon corresponding to the fractured agglomerate. 
a

NR43-N550 at $100 \%$ strain

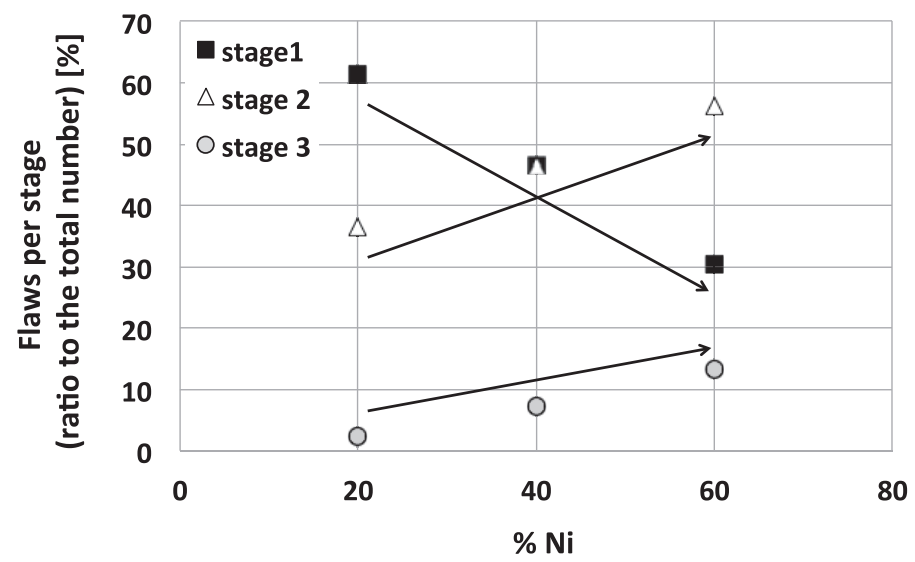

b

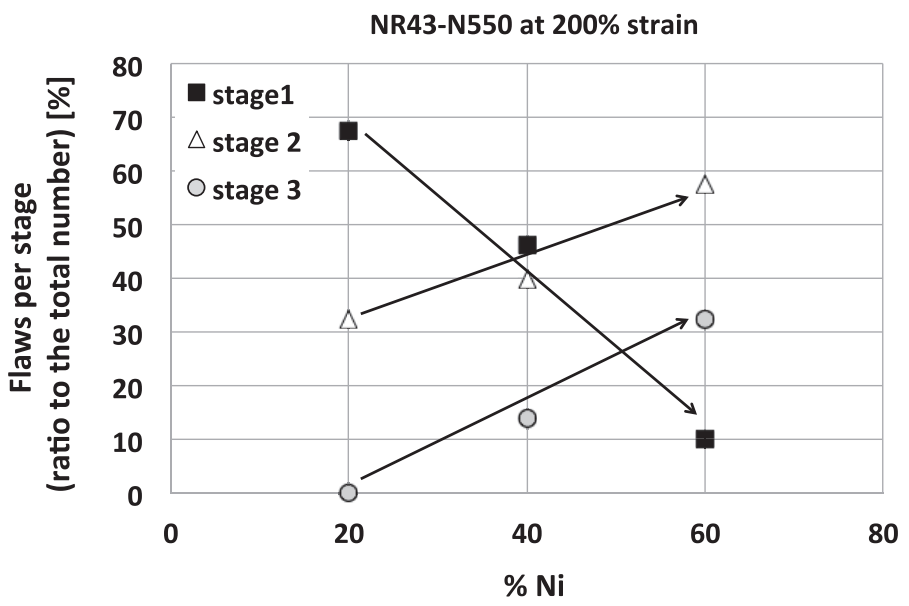

FIG. 12. - Evolution of the three stages for flaws associated with CB agglomerates in NR43-N550 with respect to the percentage of fatigue life (\% Ni): (a) at $100 \%$ strain and (b) at $200 \%$ strain.

size of the CB agglomerates for the five considered materials, including NR43-N550. As expected, this figure proves that bigger CB agglomerates shorten the fatigue life of CB-filled NR. Even if this result is logical and known for a long time for other materials (see for example, ref. 23, which studied this effect on steels), it is here clearly demonstrated in the case of CB-filled NR on the basis of a large number of observations. It definitively proves that fatigue life is more driven by the initiation phase rather than by the propagation one.

\section{FATIGUE CRACK INITIATION MECHANISM FOR ZNO INCLUSIONS}

As shown previously, $\mathrm{ZnO}$ inclusions are responsible for many initiations of cracks that in most cases do not propagate. Indeed, considering the definition of the three stages previously established, we find very few flaws in stage 3 and a majority in stage 1, regardless of the strain level.

The analysis of the SEM images shows that there are two mechanisms for stage 1: 
TABLE II

Minimum Size ( $\mathrm{S}_{\text {Min }}$ ) OF THE CB AgGlomerates, For EACH (S1, S2, S3) AS A FunCtion of the StRain AND the Percentage of FAtigue LiFe (\% Ni)

\begin{tabular}{lcccc}
\hline Strain, $\%$ & $\% \mathrm{Ni}$ & $\mathrm{s}_{\min } \mathrm{S} 1, \mu \mathrm{m}$ & $\mathrm{s}_{\min } \mathrm{S} 2, \mu \mathrm{m}$ & $\mathrm{s}_{\min } \mathrm{S} 3, \mu \mathrm{m}$ \\
\hline 60 & 20 & 5.2 & 16.5 & None \\
& 40 & 6.2 & 10.2 & None \\
& 60 & 3.0 & 3.6 & None \\
100 & 20 & 5.1 & 7.4 & 41 \\
& 40 & 4.8 & 3.5 & 12.4 \\
& 60 & 5.2 & 2.7 & 14.4 \\
& 20 & 5.0 & 3.3 & 17.3 \\
& 40 & 5.1 & 3.2 & 3.4 \\
& 60 & 2.8 & 1.5 & 2.0 \\
\hline
\end{tabular}

- fracture of the inclusion, in the case in which the internal cohesive energy of the inclusion is smaller than the bonding to the matrix (Fig. 14a) and

- debonding at one pole or at both poles, in the opposite situation (Fig. 14b,c).

$\mathrm{ZnO}$ are inclusions with low internal cohesion and low adhesion to the matrix. This explains that initiation can be easily activated (stage 1) but rarely followed by the growth of long cracks (stage 3). Figure 15 shows schematically the initiation mechanisms for stage 1 around a $\mathrm{ZnO}$ inclusion.

If one considers the average size of $\mathrm{ZnO}$ inclusions activated (stage 1) with respect to the percentage of fatigue life, it depends neither on the number of cycles nor on the strain level, as illustrated in Figure 16. This suggests that initiation does not require energy accumulation in this case. If a critical mechanical value is necessary for initiation, it is reached from $60 \%$ strain, as the size does not change with the strain level.

The size of $\mathrm{ZnO}$ inclusions may explain why, in some particular cases, they lead to crack propagation. The size of $\mathrm{ZnO}$ associated with the rare stage 3 cracks is between $14.6 \mu \mathrm{m}$ and 28.7

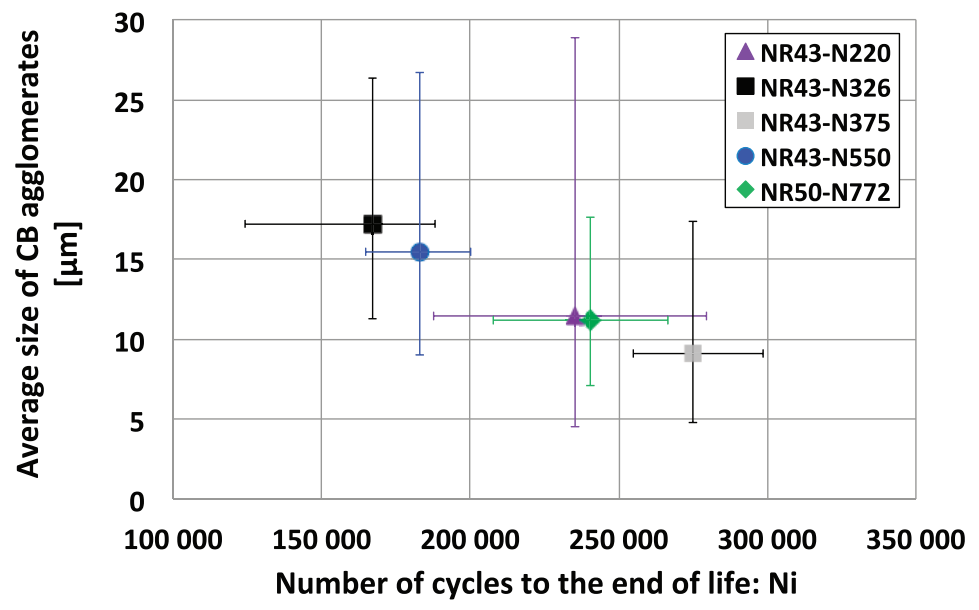

FIG. 13. - Size of the CB agglomerates on which cracks are initiated with respect to the fatigue end of life at $100 \%$ strain, for different types of $\mathrm{CB}$. 


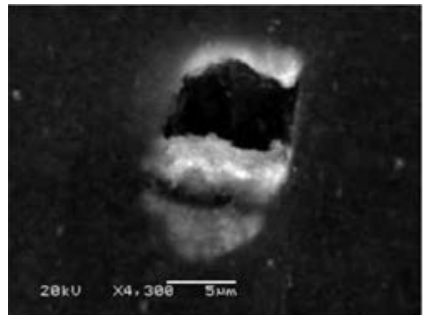

a)

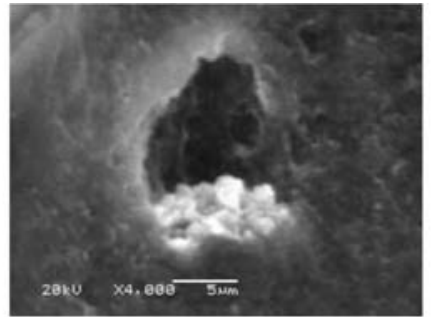

b)

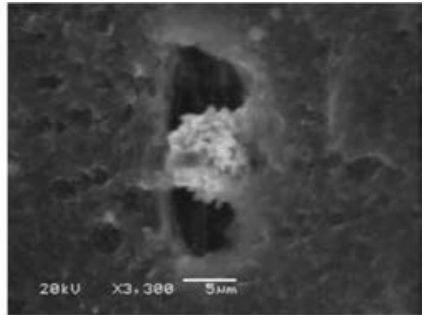

c)

FIG. 14. - The different kinds of fatigue crack initiation mechanisms around $\mathrm{ZnO}$ inclusion (stage 1): (a) fracture of the $\mathrm{ZnO}$, (b) debonding at one pole, (c) debonding at both poles.

$\mu \mathrm{m}$, which is above the average size of $\mathrm{ZnO}(7 \mu \mathrm{m})$ and close to or even higher than the average size of CB agglomerates (varying from $9 \mu \mathrm{m}$ and $17 \mu \mathrm{m}$ ). This suggests that the size of the inclusion is crucial for the propagation. However, it is surprising that some big inclusions of $\mathrm{ZnO}$ (greater than $20 \mu \mathrm{m})$ do not generate crack propagation. The shape of the inclusion, which contributes to the properties of the interface, also probably plays an important role.

\section{SUMMARY}

In the case of CB-filled NR, fatigue damage is due to multicracking at the surface of the samples initiated on geometric accidents (parting line) and on inclusions.

The number of cracks increases with the number of cycles and with the strain level.

Analysis of the initiation sites around inclusions shows that they are of various types, including two types of inclusions having a different effect on fatigue crack initiation: $\mathrm{CB}$ agglomerates and $\mathrm{ZnO}$ inclusions.

CB agglomerates have a high cohesive energy and a good adhesion to the matrix. The crack initiation mechanism involves three steps: debonding at the interface (meaning that the internal cohesion is stronger than the bonding between the inclusion and the matrix), opening on the sides, and then cracking at the surface and in the volume. Finally, at $100 \%$ strain for NR filled with

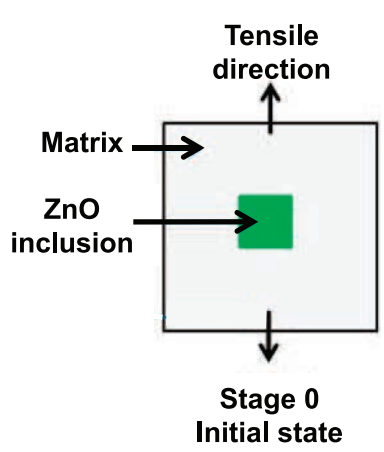

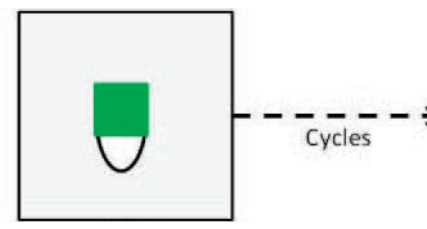

Stage 1

Debonding at the pole(s)

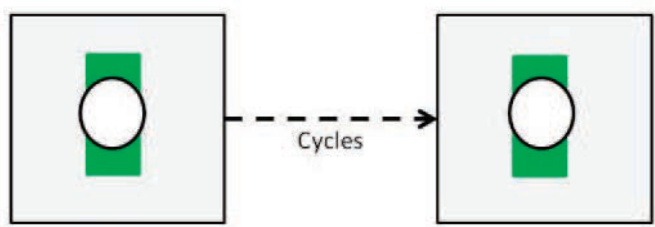

Stage 1

Inclusion fracture

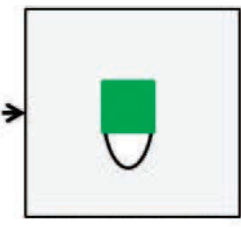

No

propagation

No

propagation

FIG. 15. - Schematic representation of fatigue crack initiation mechanism around $\mathrm{ZnO}$ inclusion (stage 1). 


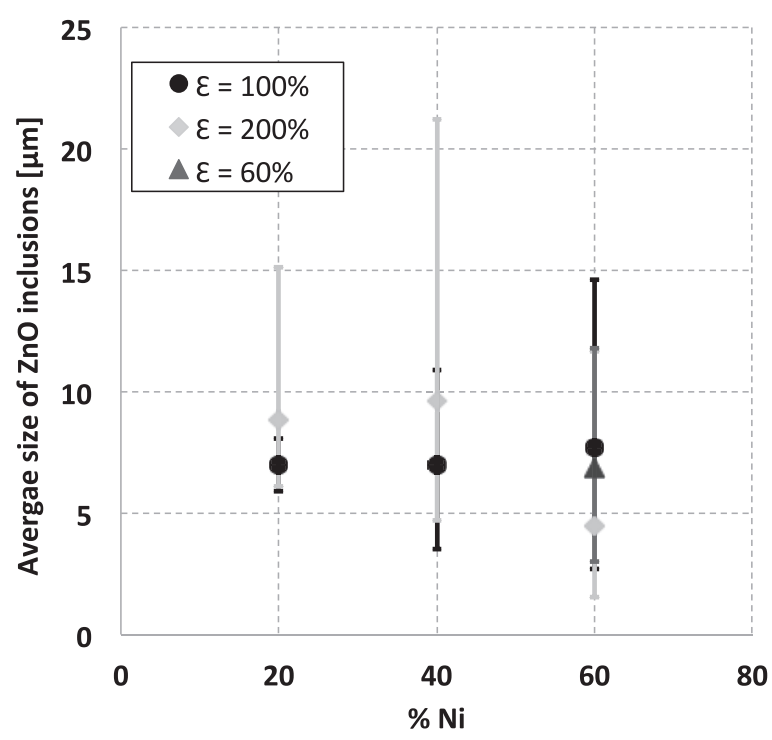

FIG. 16. - Size of the activated $\mathrm{ZnO}$ inclusions with respect to the percentage of fatigue end of life $(\% \mathrm{Ni})$ and for the three strain levels $(60 \%, 100 \%$, and $200 \%)$.

different $\mathrm{CB}$ types, fatigue life appears to be related to the average size of the $\mathrm{CB}$ agglomerates: the number of cycles leading to the end of life increases when the average agglomerate size decreases.

The internal cohesion of $\mathrm{ZnO}$ inclusions as well as their interface properties with the matrix are weak. Consequently, fatigue damage occurs by debonding or fracture of the inclusion and does not seem to be progressive. In most cases, there is no crack propagation from this kind of inclusions.

\section{ACKNOWLEDGEMENTS}

The authors would like to thank the French National Research Agency (ANR) for its financial support (ANR-2010-RMNP-010-01) and all the partners of the PROFEM project: TrelleborgVibracoustic, LMBS, GeM, UBS, and LRCCP.

\section{REFERENCES}

${ }^{1}$ W. V. Mars and A. Fatemi, Int. J. Fatigue 24, 949 (2002).

${ }^{2}$ A. N. Gent, P. B. Lindley, and A. G. Thomas, J. Appl. Polym. Sci. 8, 455 (1964).

${ }^{3}$ J. R. Beatty, RubBer CHEM. TECHNOL. 37, 1341 (1964).

${ }^{4}$ A. A. Griffith, Phil. Trans. R. Soc. London A Math. Phys. Eng. Sci. 221, 582 (1921).

${ }^{5}$ S. Cadwell, R. Merrill, C. Sloman, and F. Yost, Ind. Eng. Chem. 12, 19 (1940).

${ }^{6}$ G. J. Lake, RubBer CHEM. TeChNOL. 68, 435 (1995).

${ }^{7}$ W. V. Mars and A. Fatemi, Rubber Chem. TeChNol. 77, 391 (2004).

${ }^{8}$ N. Saintier, G. Cailletaud, and R. Piques, Int. J. Fatigue 28, 61 (2006).

${ }^{9}$ A. Bennani, “Title,” Ph.D. thesis [in French], École Nationale Supérieure des Mines de Paris, 2006.

${ }^{10}$ K. Le Gorju Jago, "Fatigue Life of Rubber Components: 3D Damage Evolution from X-ray Computed Microtomography," in Constitutive Models for Rubber V, A. Boukamel, L. Laiarinandrasana; S. Méo; and E. Verron, Eds., Paris, Taylor \& Francis/Balkema, 2007, pp 173-177. 
${ }^{11}$ J. B. Le Cam, B. Huneau, and E. Verron, Int. J. Fatigue 52, 82 (2013).

${ }^{12}$ B. Huneau, I. Masquelier, Y. Marco, O. Brzokewicz, and P. Charrier, "Fatigue Damage in Carbon Black Filled Natural Rubber Investigated by X-ray Microtomography and Scanning Electron Microscopy," in Constitutive Models for Rubber VIII, N. Gil-Negrete and A. Alonso, Eds., CRC Press-Taylor \& Francis, San Sebastian, Spain, 2013, pp 393-398.

${ }^{13}$ I. Masquelier, “Title,” Ph.D. thesis [in French], Université Bretagne Occidentale, 2014.

${ }^{14}$ K. Le Gorju Jago, RubBer CHEM. TeCHNOL. 85, 387 (2012).

${ }^{15}$ V. Le Saux, Y. Marco, S. Calloch, and P. Charrier, Polym. Eng. Sci. 51, 1253 (2011).

${ }^{16}$ A. K. Bhowmick, G. B. Nando, S. Basu, and S. K. De, Rubber ChEM. Technol. 53, 327 (1980).

${ }^{17}$ J. B. Le Cam, B. Huneau, E. Verron, and L. Gornet, Macromolecules 37, 5011 (2004).

${ }^{18}$ S. V. Hainsworth, Polym. Test. 26, 60 (2007).

${ }^{19}$ S. Beurrot, B. Huneau, and E. Verron, J. Appl. Polym. Sci. 117, 1260 (2010).

${ }^{20}$ M. Flamm, J. Spreckels, T. Steinweger, and U. Weltin, Int. J. Fatigue 33, 1189 (2011).

${ }^{21}$ E. Ostoja-Kuczynski, P. Charrier, E. Verron, G. Marckmann, L. Gornet, and G. Chagnon, "Crack Initiation in Filled Natural Rubber: Experimental Database and Macroscopic Observations," in Constitutive Models for Rubber III, J. Busfield and A. Muhr, Eds., Taylor \& Francis, London, 2003, pp 41-47.

${ }^{22}$ V. Collin and E. Peuvrel-Disdier, Elastomery 9, 9 (2005).

${ }^{23}$ Y. Murakami, S. Kodama, and S. Konuma, Int. J. Fatigue 11, (1989). 